\title{
Research Article \\ Simplified Printed Log-Periodic Dipole Array Antenna Fed by CBCPW
}

\author{
Guohua Zhai, ${ }^{1}$ Yong Cheng, ${ }^{2}$ Qiuyan Yin, ${ }^{1}$ Shouzheng Zhu, ${ }^{1}$ and Jianjun Gao ${ }^{1}$ \\ ${ }^{1}$ School of Information and Science Technology, East China Normal University, Shanghai 200241, China \\ ${ }^{2}$ Shanghai Normal University, Tianhua College, Shanghai 201815, China \\ Correspondence should be addressed to Guohua Zhai; ghuzhai@gmail.com
}

Received 29 August 2013; Accepted 3 September 2013

Academic Editor: Xiao Ping Chen

Copyright (c) 2013 Guohua Zhai et al. This is an open access article distributed under the Creative Commons Attribution License, which permits unrestricted use, distribution, and reproduction in any medium, provided the original work is properly cited.

\begin{abstract}
A novel simple approach for designing single-layer printed log-periodic dipole array (PLPDA) antenna fed by conductor-backed coplanar waveguide (CBCPW) is presented. The PLPDA antenna has the merits of wide bandwidth, low profile, and stable radiation pattern, which should be fed by the balanced line. The balanced line, created by the geometric features of the CBCPW, provides a balun with a considerably wide bandwidth with low profile, broad bandwidth, low insertion loss, and so forth. The prototype of the proposed CBCPW-fed printed log-periodic array at Ka band is designed and fabricated; the measured data are in good agreement with the simulated results.
\end{abstract}

\section{Introduction}

As an end-fire type antenna, the log-periodic dipole array (LPDA) antenna has the merits of fixed peak radiation and stable radiation pattern within the operating frequency, so it is one of the candidates for the modern wireless communications system. Numerous studies have been documented on the design method and the performance of the conventional LPDA antenna since the 1960s [1-7].

In order to achieve low profile, printed log-periodic dipole arrays (PLPDA) antenna was proposed based on the printed integrated board (PCB) technology [8-14]. Practically, the performance of the PLPDA antenna is determined by the feeding network. At an early stage, the PLPDA antenna was designed on a piece of the double-layer printed circuit board [8-11], but it increases the fabrication cost and limits the productivity. Then, the fabrication cost is reduced by using single-layer printed circuit, so the single-layer PLPDA antennas with two coaxial cables as the feeding network were proposed [12, 13]. However, soldering coaxial cables bring significant fabrication error and misalignment, especially at millimeter-wave frequencies. A single-layer slot PLPDA antenna fed by coplanar waveguide (CPW) was proposed [14], the CPW transmission line has been widely used in the monolithic millimeter-wave integrated circuits (MMIC), but this transmission line suffers from high insertion loss in the transition, radiation loss, serious crosstalk, and lower power handling capability because of its open structure.

Recently, the substrate integrated circuits (SIC) concept, in particular substrate integrated waveguide (SIW) technique, has been demonstrated as a promising scheme for low-cost, small size, relatively high power, low radiation loss, and high-density integrated microwave and millimeter-wave components and systems, which has been widely used in the low profile antenna design [15-18]. And the substrate integrated PLPDA antenna is proposed [19]. However, this new feeding network is larger, especially at lower frequency. And the printed dipoles of the PLPDA antenna mentioned above are all cross-symmetrically spaced along the parallel feed lines on both sides of the dielectric substrate. So, it necessitates two sides of the substrate to be etched.

In this paper, a simplified PLPDA antenna fed by CBCPW is presented, which can be called CBCPW PLPDA antenna. The geometry of the proposed antenna is shown in Figure 1. It has advantages of low profile, broadband, light weight, and ease of integration to the planar circuits. The entire structure is fabricated on a piece of PCB. And all the printed dipoles and 


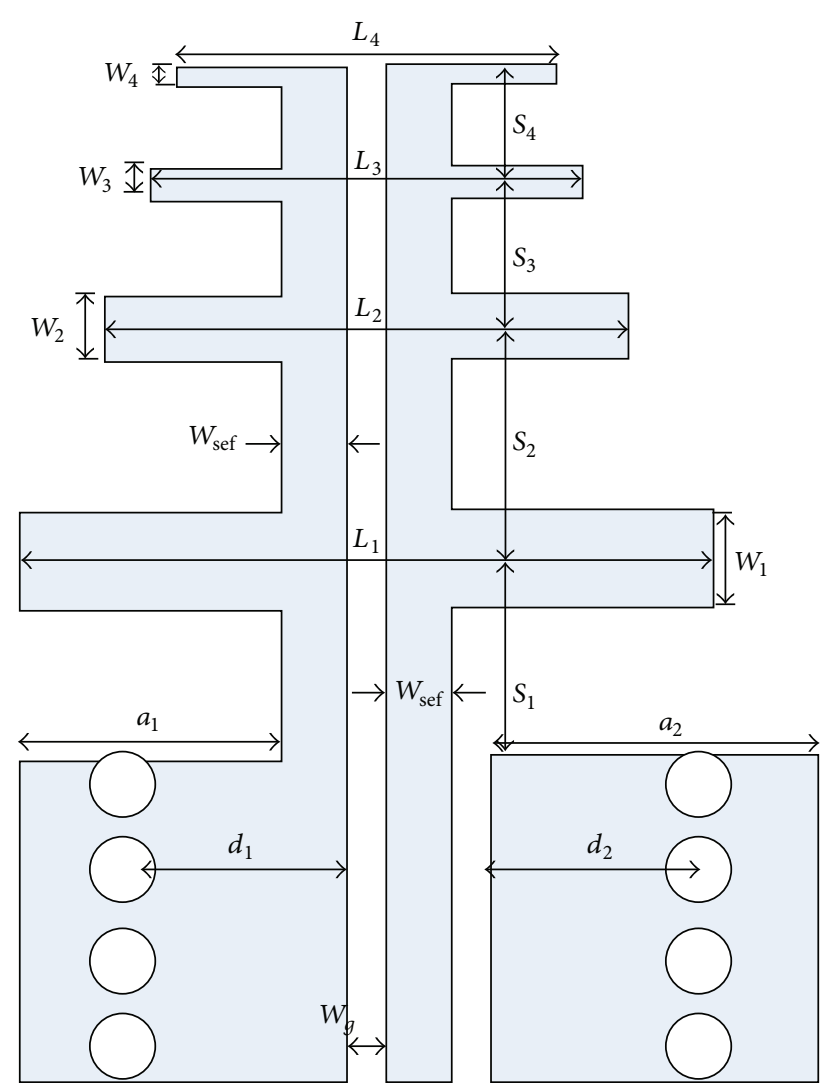

(a) Top layer

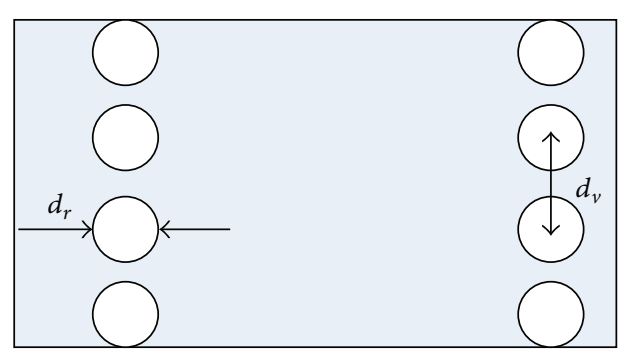

(b) Bottom layer

FIGURE 1: Geometry of the proposed CBCPW PLPDA antenna.

the feed lines of the PLPDA antenna are printed on only one side of the dielectric substrate. Therefore, this novel antenna alleviates the deign complexity, reduces the cost, and can be easy to integrate with MMIC.

\section{CBCPW PLPDA Antenna Design}

For the proposed antenna design, the $50 \Omega \mathrm{CBCPW}$ is directly transited to the two parallel transmission strip lines, along which the dipole elements are symmetrically distributed on the top metallic layer of the substrate. One parallel line is connected to the central line of the CBCPW, and the other one is connected to one side of the ground on the top side of the CBCPW. The bottom ground of the CBCPW is etched on the bottom layer of the substrate; the top and the bottom metallic layers of the CBCPW are connected by the metallic vias to balance the transmission line.

Figure 2 depicts the dominant mode in the cross-section of the $\mathrm{CBCPW}$, which clearly shows the $180^{\circ}$ phase difference between the central line and the ground of the CBCPW. Therefore, the structure of $\mathrm{CBCPW}$ can provide a good performance over a fairly wide bandwidth and perform a good balun line for the feeding network of the single-layer PLPDA antenna.

The electromagnetic power excited by the feeder is transmitted from CBCPW to the dipole elements along the

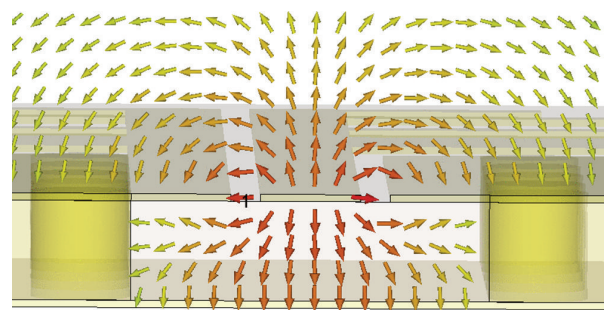

FIGURE 2: Simulated electric field distribution of the dominant mode in the cross-section of the CBCPW.

two parallel transmission strips line, which is shown in Figure 3. And the parallel transmission strip lines acts as a transformer between the CBCPW and the PLPDA antenna; it also performs as the balun. Therefore, the currents of the dipole elements on the left side are in phase with those printed in the opposite direction on the right side of the substrate, which results in mono-directional radiation towards the shorter elements.

The parameters of the proposed antenna are determined by the modified Carrel method [2], including an effective relative dielectric permittivity in computations to dipole lengths and spacing. According to the principle of the PLPDA antenna, the optimum values of the parameters $L_{n}, S_{n}$, and $W_{n}$ 

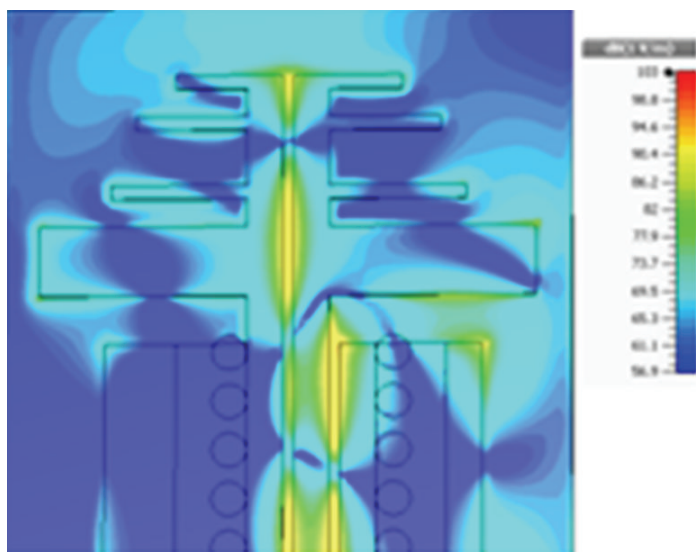

FIGURE 3: Simulated electric field density distributions of the proposed antenna $(35 \mathrm{GHz})$.

can be determined by the scale factor $\tau$ and spacing factor $\sigma$ as given by $[7,8]$

$$
\begin{gathered}
\tau=\frac{L_{(n+1)}}{L_{n}}=\frac{W_{n+1}}{W_{n}}, \\
\sigma=\frac{1-\tau}{4 * \tan \alpha}=\frac{S_{n}}{4 * L_{n}} .
\end{gathered}
$$

The value of the structure bandwidth $B_{s}=B \times B_{\mathrm{ar}}$, where $B$ is the desired bandwidth ratio and $B_{\mathrm{ar}}$ is the bandwidth for the active region given by $[7,8]$

$$
B_{\mathrm{ar}}=1.1+7.7(1-\tau)^{2} \cot \alpha .
$$

The number of the dipole elements $N$ is obtained from $[7,8]$

$$
N=1+\frac{\log B_{s}}{\log (1 / \tau)} .
$$

The actual half-length of the largest dipole element adjacent to the feed point is given by $[7,8]$

$$
\begin{gathered}
L_{1}=\frac{\lambda_{\text {eff } \min }}{2}=\frac{c}{2 f_{\max \sqrt{\varepsilon_{\text {eff }}}}}, \\
\varepsilon_{\text {eff }}=\frac{\varepsilon_{r}+1}{2}+\frac{\varepsilon_{r}+1}{2} \frac{1}{\sqrt{1+12 h / W_{1}}},
\end{gathered}
$$

where $\lambda_{\text {eff min }}$ is the shortest effective working wavelength, $\varepsilon_{\text {eff }}$ is the effective dielectric permittivity, $\varepsilon_{r}$ is the dielectric constant, $h$ is the substrate thickness, and $W_{1}$ is the width of the first dipole element.

Hence, the parameters of this structure to be tuned are $\tau, \sigma, W_{1}, S_{1}$, and $W_{\text {sef }}$, which can be optimized to further enhance the performance. In this paper, the proposed CBCPW PLPDA antenna is designed at $25-40 \mathrm{GHz}$, which is printed on one side of a $0.5 \mathrm{~mm}$ thick FR- 4 substrate with the permittivity of 4.3 . The size parameters of the $50 \Omega \mathrm{CBCPW}$ can be first decided, and then the length of the longest dipole $L_{1}$ can be calculated from (5). Then, the length of the other elements can be calculated from (1); meanwhile, the width of
TABLE 1: Dimensions of CBCPW PLPDA antenna.

\begin{tabular}{lc}
\hline Symbol & Quantity \\
\hline$W_{1}$ & 1 \\
$W_{4}$ & 0.2 \\
$L_{3}$ & 4.3 \\
$S_{2}$ & 1 \\
$W_{\text {sef }}$ & 0.5 \\
$d_{1}$ & 1 \\
$a_{2}$ & 2 \\
$W_{2}$ & 0.2 \\
$L_{1}$ & 7 \\
$L_{4}$ & 3.2 \\
$S_{3}$ & 1 \\
$W_{g}$ & 0.15 \\
$d_{2}$ & 1 \\
$d_{r}$ & 0.25 \\
$W_{3}$ & 0.2 \\
$L_{2}$ & 5 \\
$S_{1}$ & 1.2 \\
$S_{4}$ & 0.6 \\
$h$ & 0.5 \\
$a_{1}$ & 2 \\
$d_{v}$ & 0.8 \\
\hline Unit: $\mathrm{mm}$. &
\end{tabular}

the longest dipole $W_{1}$, the feed line $W_{\text {sef }}$, and the distance from the feed point to the first dipole $S_{1}$ can be optimized by the full-wave simulation software HFSS. The width of the other elements $W_{n}$ and the spacing between the elements $S_{n}$ can be obtained from (1) and (2). After optimization with the fullwave simulation software HFSS, the dipole element $N$ is 4 , and the other geometry parameters of the proposed antenna are shown in Table 1.

\section{Experimental Results}

The proposed PLPDA antenna at $25 \mathrm{GHz}-40 \mathrm{GHz}$ is designed, fabricated, and measured. The measured and simulated return losses of the proposed PLPDA antenna are shown in Figure 4. All of the measured results shown below include the losses of $50 \Omega \mathrm{CBCPW}$ insertion loss, the $2.4 \mathrm{~mm}$ to $3.5 \mathrm{~mm}$ adapters, and the SMA connectors. It can be seen that the $\left|S_{11}\right|$ is below $-10 \mathrm{~dB}$ from $28 \mathrm{GHz}$ to $40 \mathrm{GHz}$. There is a little difference in the center frequency between measurement and simulation, which may be brought by the fabrication errors and frequency dependent dielectric permittivity without consideration in the simulation.

Figure 5 shows the measured antenna gain of the proposed antennas. The peak antenna gain of the CBCPW PLPDA antenna is $4.3 \mathrm{dBi}$ at $34 \mathrm{GHz}$. The measured antenna gain of the CBCPW PLPDA antenna is less than that of the simulated results, which maybe brought by the losses of the SMA connector and the increasing substrate loss of the antenna at millimeter-waves.

The far-field radiation pattern of the proposed antenna is measured which shows the similar characteristics across 


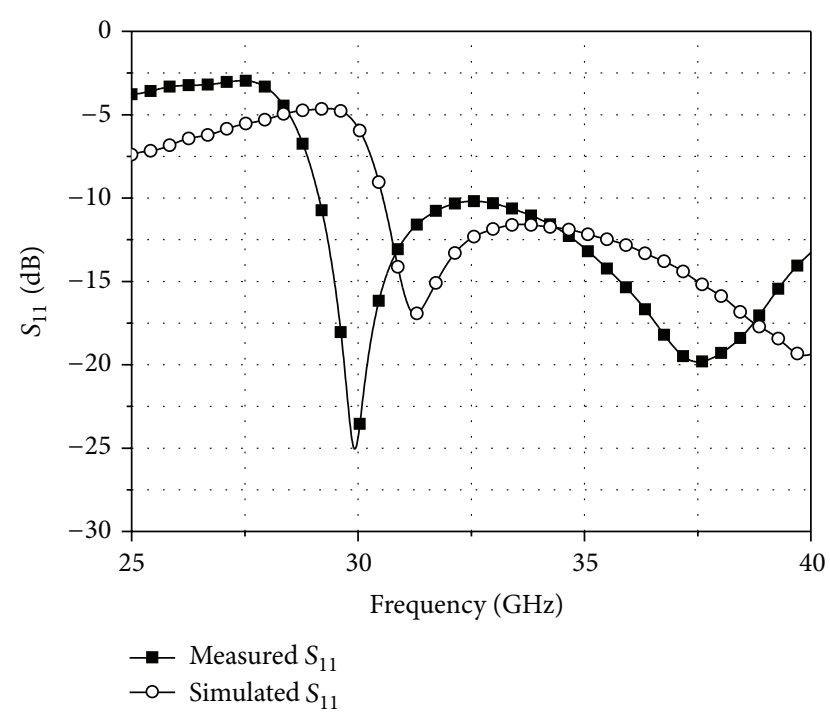

FIGURE 4: Simulated and measured return loss of the proposed antenna.

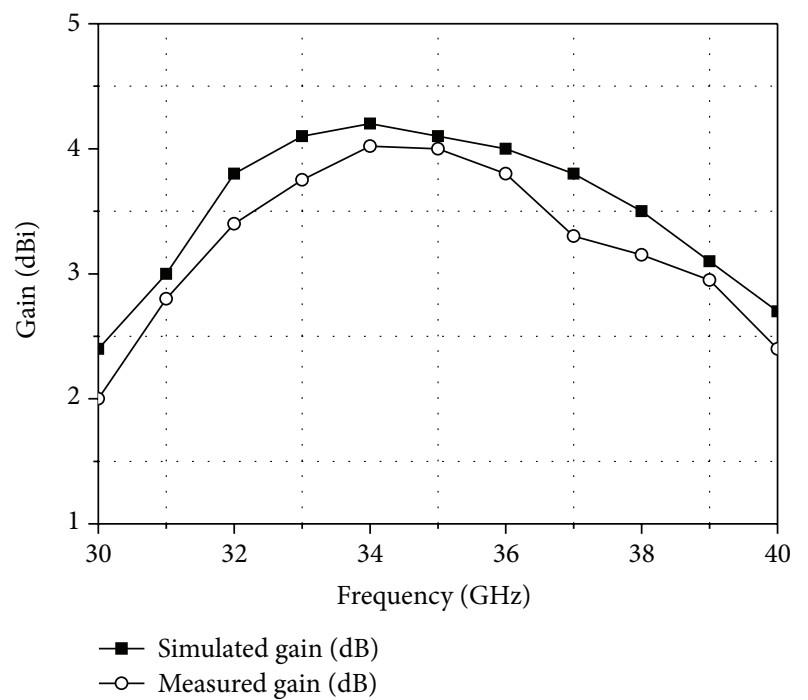

Figure 5: Simulated and measured gain of the proposed antenna.

the operating bandwidth. And for brevity, only the $35 \mathrm{GHz}$ radiation patterns are shown in Figure 6, which shows that the front-to-back ratio is larger than $15 \mathrm{~dB}$, and the 3D farfield pattern of the proposed antenna at $35 \mathrm{GHz}$ is given in Figure 7, which shows that the proposed CBCPW PLPDA antenna has a good end-fire radiation characteristic.

\section{Conclusion}

In this paper, a low profile, low-cost, and simple design PLPDA antenna fed by CBCPW has been proposed. The prototype for the proposed CPW PLPDA antenna has been designed, fabricated, and measured. It is simple to make a prototype by using standard PCB fabrication technique. Because the feeding network does not require additional devices, such as a T-junction, power divider, or microstripto-coplanar strip line (CPS) balun, this antenna is simple

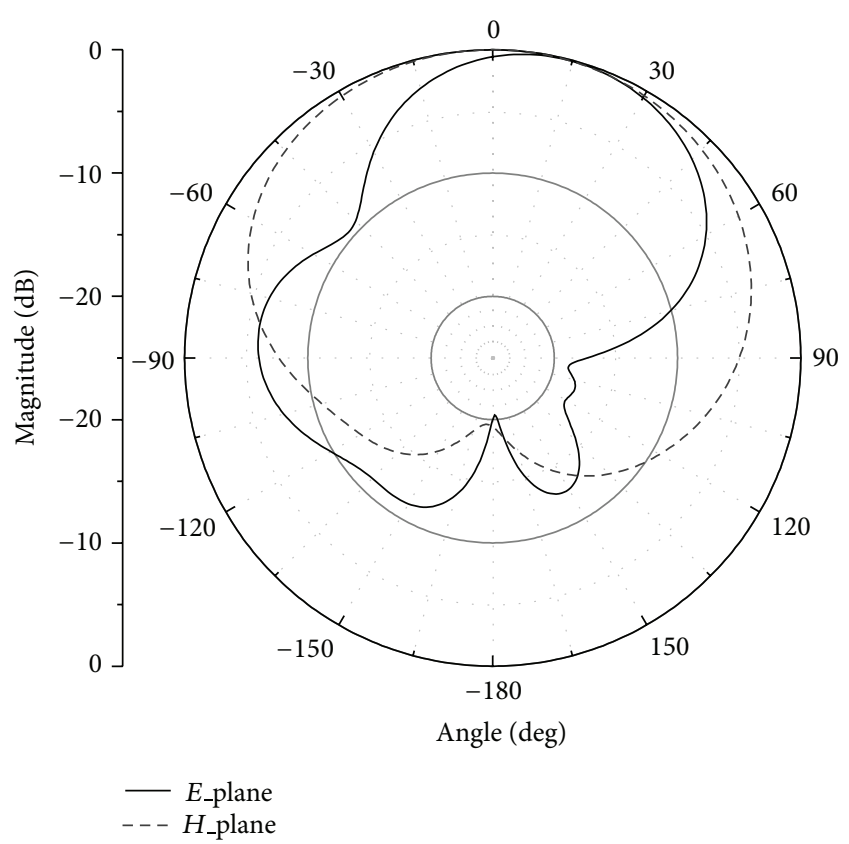

FIGURE 6: Measured far-field of the proposed PLPDA antenna at $35 \mathrm{GHz}$.

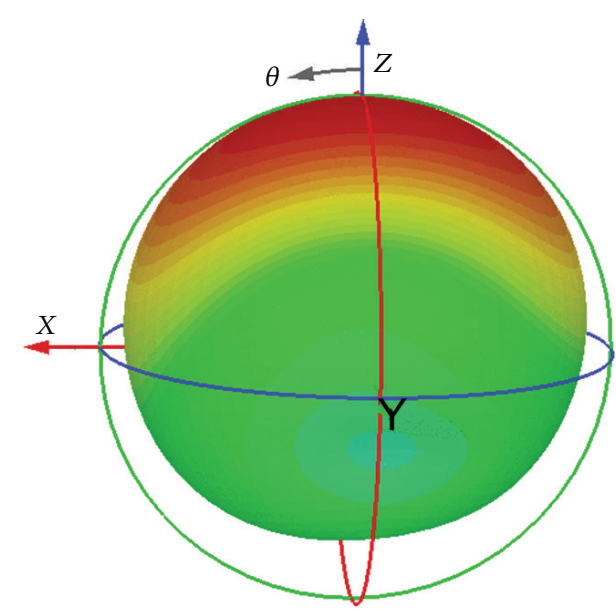

FIgURE 7: Simulated 3D far-field of the proposed PLPDA antenna at $35 \mathrm{GHz}$.

to design. The low profile antenna structure results in ease of integration into planar microwave and millimeter-wave wireless communications systems. The proposed concept reduces the size and design complexity of the antenna. Radiation pattern and antenna gain of the CBCPW PLPDA antenna are presented and studied. The advantages such as low cost, broadband, compact, and simplicity are verified by the experiment.

\section{Acknowledgments}

This work was supported in part by NSFC under Grant 61201069, in part by Research Fund for the Doctoral Program of Higher Education of China under Grant 20120076120035, 
and in part by Open-Foundation of State Key Laboratory of Millimeter-Waves under Grate 201308.

\section{References}

[1] D. Isbell, "Log periodic dipole arrays," IRE Transactions on Antennas and Propagation, vol. 8, no. 3, pp. 260-267, 1960.

[2] R. Carrel, "The design of the log-periodic dipole antenna," IRE International Conventional Record, vol. 9, part 1, pp. 61-75, 1961.

[3] J. Carr, "Some variations in log-periodic antenna structures," IRE Transactions on Antennas and Propagation, vol. 9, no. 2, pp. 229-230, 1961.

[4] R. Mittra and K. E. Jones, "Theoretical brillouin (k- $\beta$ ) diagrams for monopole and dipole arrays and their applications to log-periodic antennas," IEEE Transactions on Antennas and Propagation, vol. 51, no. 3, pp. 533-540, 1964.

[5] N. Barbano, "Waveguide-fed log periodic antennas," IRE Transactions on Antennas and Propagation, vol. 16, no. 3, pp. 357-358, 1968.

[6] J. Wolter, "Solution of Maxwell's equations for log-periodic dipole antennas," IEEE Transactions on Antennas and Propagation, vol. 18, no. 6, pp. 734-741, 1970.

[7] A. B. Constantine, Antenna Theory: Analysis and Design, WileyInterscience, New York, NY, USA, 2005.

[8] C. K. Campbell, I. Traboulay, M. S. Suuthers, and H. Kneve, "Design of a stripline log-periodic dipole antenna," IEEE Transactions on Antennas and Propagation, vol. 25, no. 5, pp. 718-721, 1977.

[9] P. Alakananda and G. Inderjeet, "An analysis of log periodic antenna with printed dipoles," IEEE Transactions on Antennas and Propagation, vol. 29, no. 2, pp. 114-119, 1981.

[10] R. R. Pantoja, A. R. Sapienza, and F. C. Medeiros Filho, "A microwave printed planar log-periodic dipole array antenna," IEEE Transactions on Antennas and Propagation, vol. 35, no. 10, pp. 1176-1178, 1987.

[11] F. Merli, J.-F. Zurcher, A. Freni, and A. K. Skrivervik, "Analysis, design and realization of a novel directive ultrawideband antenna," IEEE Transactions on Antennas and Propagation, vol. 57, no. 11, pp. 3458-3466, 2009.

[12] X. Liang and Y. W. M. Chia, "New precision wideband direction finding antenna," IEE Proceedings: Microwaves, Antennas and Propagation, vol. 148, no. 6, pp. 363-364, 2001.

[13] D. E. Anagnostou, J. Papapolymerou, M. M. Tentzeris, and C. G. Christodoulou, "A printed Log-Periodic Koch-Dipole Array (LPKDA)," IEEE Antennas and Wireless Propagation Letters, vol. 7, pp. 456-460, 2008.

[14] S. H. Kim, J. H. Choi, J. W. Baik, and Y. S. Kim, "CPW-fed logperiodic dumb-bell slot antenna array," Electronics Letters, vol. 42, no. 8, pp. 436-438, 2006.

[15] G. Q. Luo, Z. F. Hu, W. J. Li, X. H. Zhang, L. L. Sun, and J. F. Zheng, "Bandwidth-enhanced low-profile cavity-backed slot antenna by using hybrid SIW cavity modes," IEEE Transactions on Antennas and Propagation, vol. 60, no. 4, pp. 1698-1704, 2012.

[16] G. Q. Luo, Z. F. Hu, Y. Liang, L. Y. Yu, and L. L. Sun, "Development of low profile cavity backed crossed slot antennas for planar integration," IEEE Transactions on Antennas and Propagation, vol. 57, no. 10, pp. 2972-2979, 2009.

[17] G. Q. Luo, Z. F. Hu, L. X. Dong, and L. L. Sun, "Planar slot antenna backed by substrate integrated waveguide cavity," IEEE Antennas and Wireless Propagation Letters, vol. 7, pp. 236-239, 2008.
[18] G. Q. Luo, L. L. Sun, and L. X. Dong, "Single probe fed cavity backed circularly polarized antenna," Microwave and Optical Technology Letters, vol. 50, no. 11, pp. 2996-2998, 2008.

[19] G. H. Zhai, W. Hong, K. Wu, and Z. Q. Kuai, "Wideband substrate integrated printed log-periodic dipole array antenna," IET Microwaves, Antennas and Propagation, vol. 4, no. 7, pp. 899-905, 2010. 

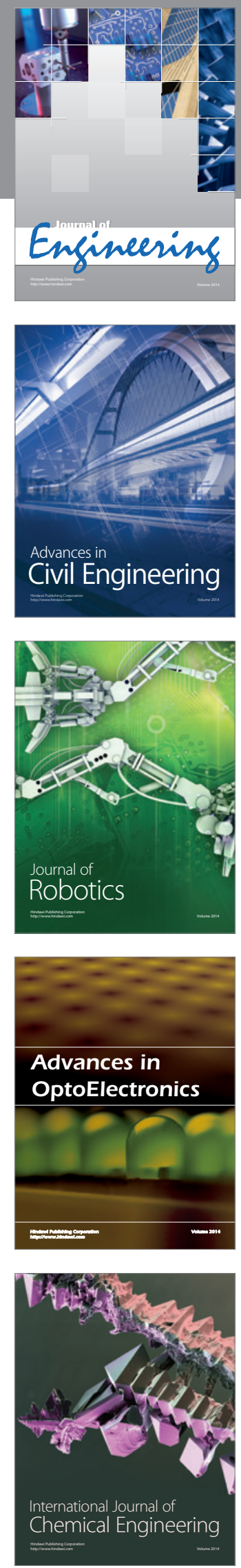

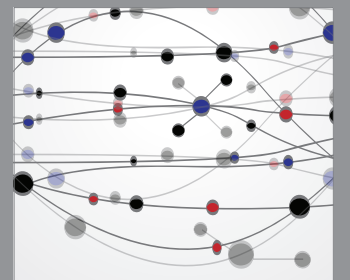

The Scientific World Journal
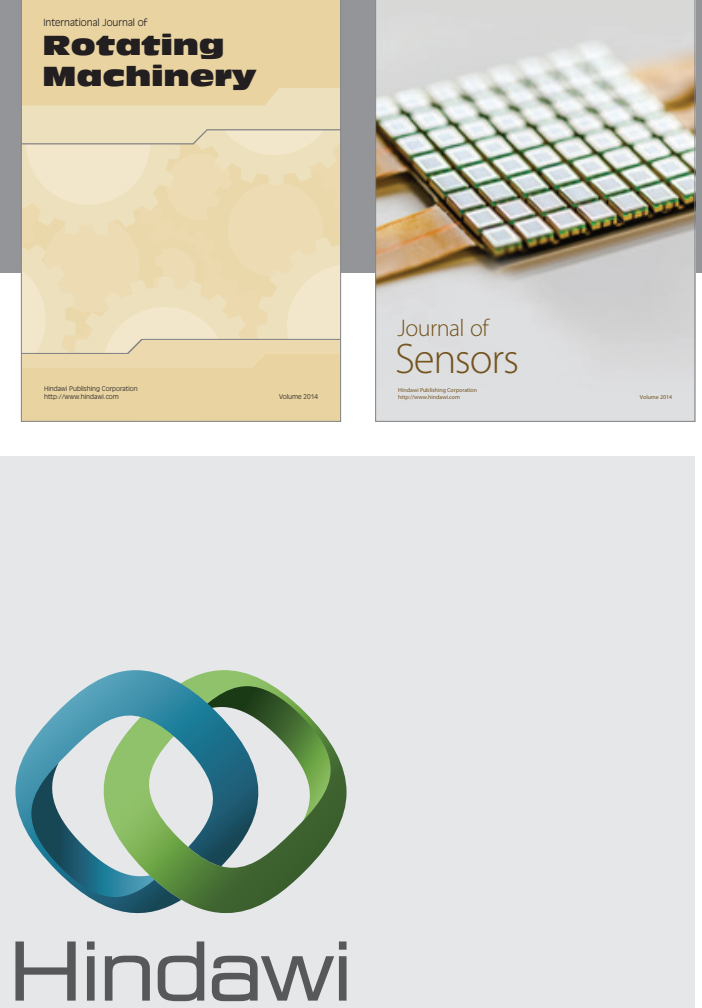

Submit your manuscripts at http://www.hindawi.com
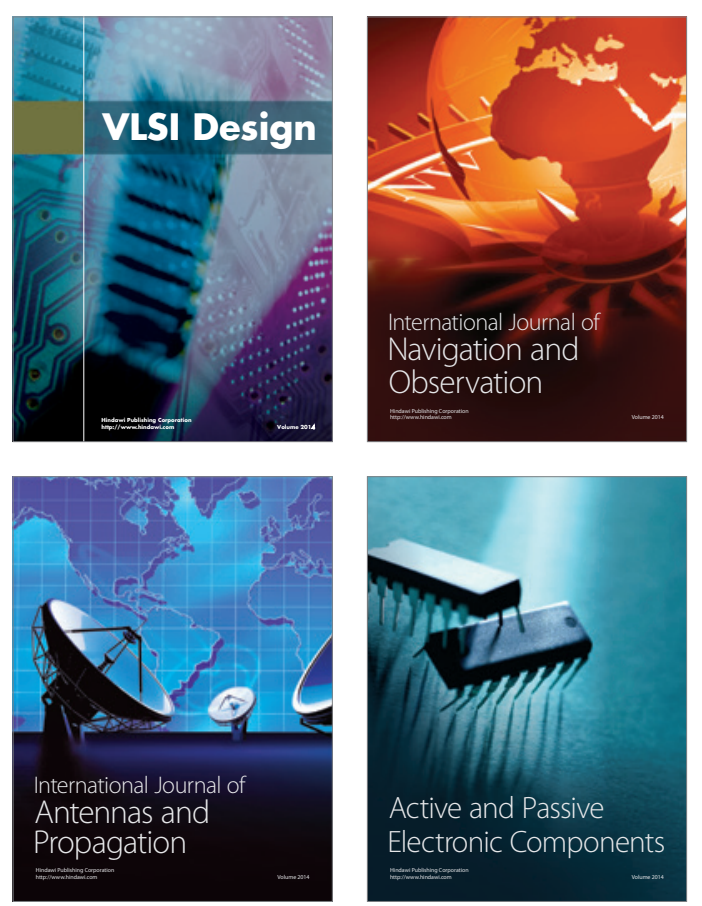
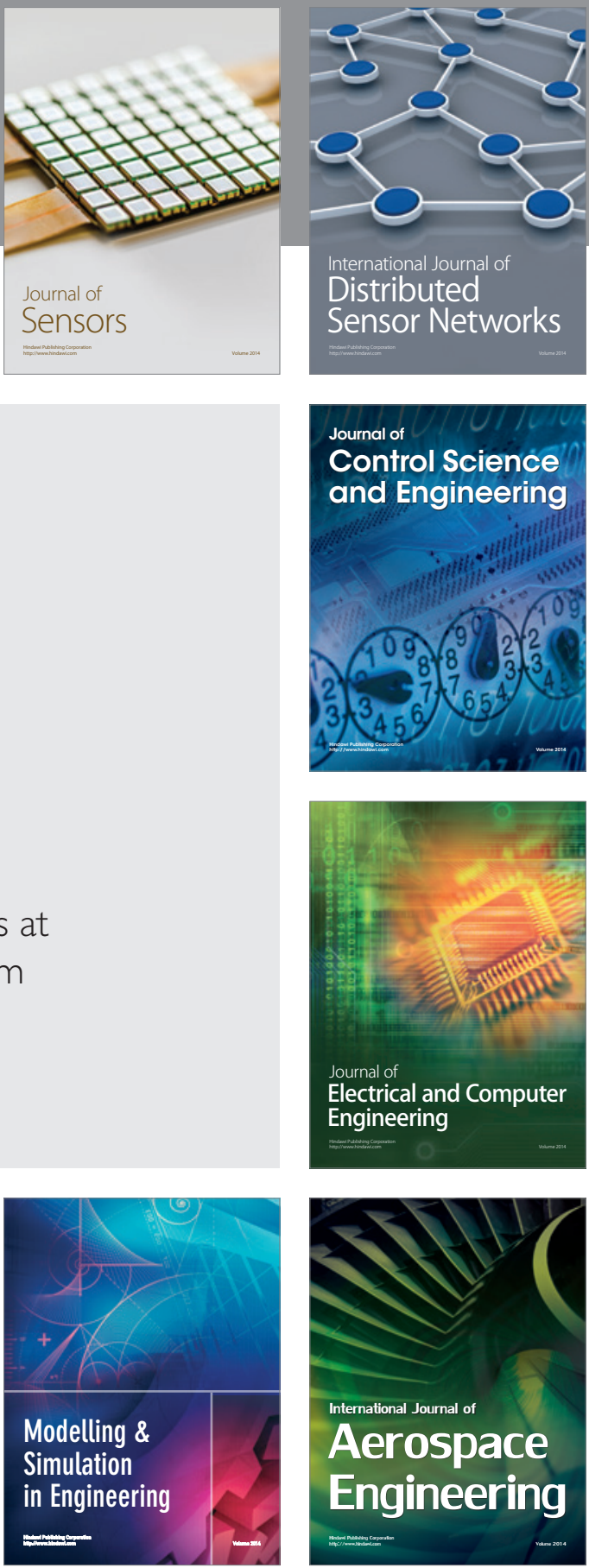

Journal of

Control Science

and Engineering
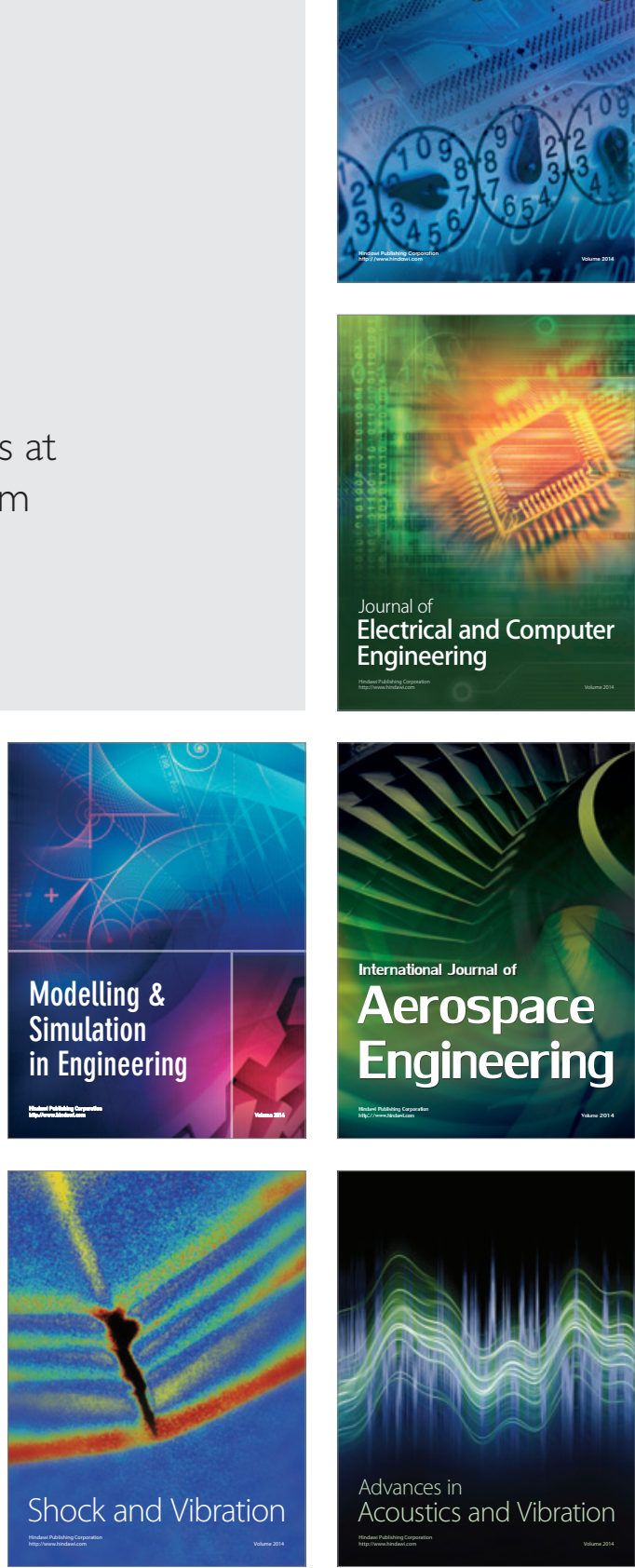